\title{
Síndrome del creciente temporal. Reporte de un caso y revisión de la literatura
}

\author{
Victoria Mery $C^{\mathbf{a}}$, Patricio Mellado $\mathbf{T}^{\mathbf{1}}$, Raúl Valenzuela \\ $\mathrm{M}^{1}$, Cristián Luco $\mathrm{F}^{1}$, Isidro Huete $\mathrm{L}^{2}$. \\ Temporal crescent syndrome. Report \\ of one case
}

The temporal crescent syndrome or half-moon syndrome is a rare mono ocular retrochiasmatic visual field defect that can be correlated to a lesion along the contralateral parieto-occipital sulcus. This field defect may be missed in automated perimetry. We report a 45 years old man, consulting for sudden loss of the peripheral temporal field in his right eye. The magnetic resonance imaging and the spectroscopy studies confirmed an ischemic lesion on the left anterior occipital cortex. Control imaging studies six months later did not show changes in the lesion (Rev Méd Chile 2004; 132: 1523-6).

(Key Words: Temporal crescent syndrome; Visual acuity; Visual fields)

Recibido el 4 de mayo, 2004. Aceptado el 17 de agosto, 2004.

${ }_{1}^{1}$ Departamentos de Neurología y ${ }^{2}$ Radiología, Facultad de Medicina, Hospital Clínico de la Pontificia Universidad Católica de Chile. Santiago de Chile.

Interna de Medicina

La as lesiones retroquiasmáticas de la vía óptica afectan el campo visual de forma homónima (hemianopsia o cuadrantopsia homónima contralateral) y las lesiones prequiasmáticas (retinianas o del nervio óptico), son las causantes de la mayoría de los defectos monoculares. La excepción a esta regla es el síndrome del creciente temporal, que corresponde a una alteración infrecuente del campo visual, caracterizado por un defecto temporal monocular lateral en forma de media luna y se correlaciona con una lesión anterior en el lóbulo occipital contralateral. Comunicamos el caso de un paciente con un defecto monocular del creciente temporal derecho.

Correspondencia a: Dr. Patricio Mellado T. Marcoleta 347, Santiago, Chile. Fax: 56-2-632-6221. E-mail: pmellado@med.puc.cl

\section{CASO CLÍNICO}

Hombre de 45 años, diestro, sin antecedentes mórbidos. Consultó debido a que notó, súbitamente, dificultad para detectar los objetos que aparecían por la derecha y atrás de su cabeza. En su evaluación clínica tenía un examen general y neurológico normal, con una agudeza visual de 20/20 en cada ojo y pupilas simétricas sin defectos aferentes ni eferentes. Sin embargo, en el estudio de campimetría por confrontación se evidenció un defecto de campo temporal lateral del ojo derecho. El perímetro de Goldmann del ojo derecho mostraba un defecto periférico que se extendía entre los $60^{\circ}$ y $90^{\circ}$ del meridiano horizontal (Figura 1). El campo visual del ojo izquierdo era normal, extendiéndose a $90^{\circ}$ temporal y a $60^{\circ}$ 
FiguRA 1. Campos visuales con perímetro de Goldmann. El ojo derecho muestra un defecto periférico que se extiende entre los $60^{\circ}$ y $90^{\circ}$ del meridiano horizontal. El campo visual del ojo izquierdo es normal, extendiéndose a $90^{\circ}$ temporal y a $60^{\circ}$ nasal.

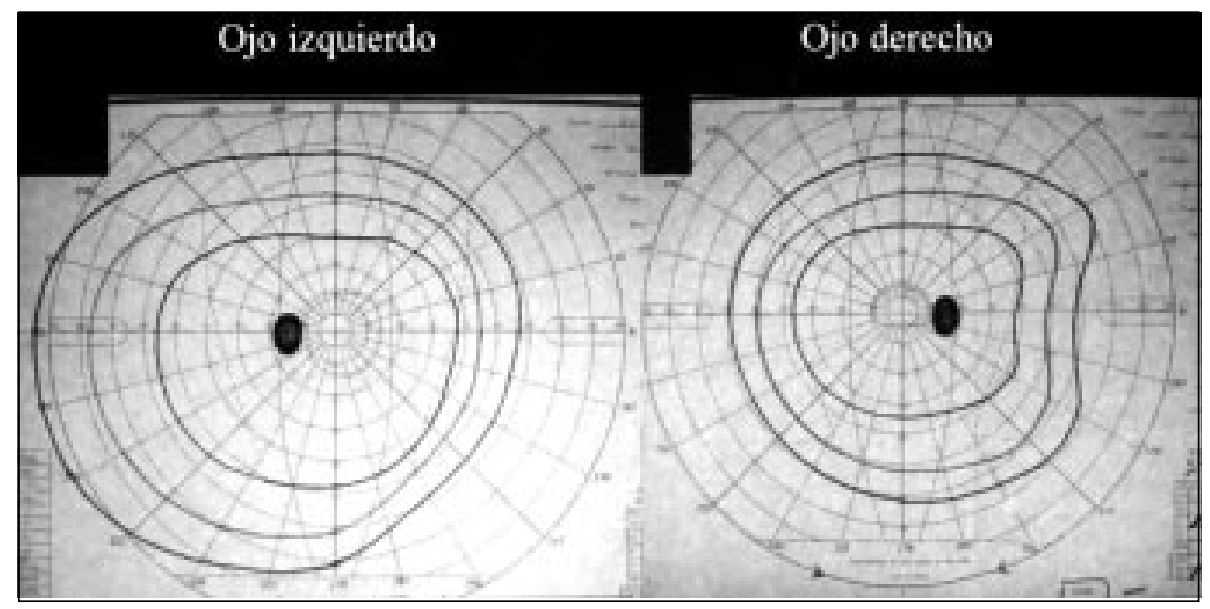

nasal (Figura 1). El fondo de ojo era normal en ambos ojos. La resonancia magnética $(\mathrm{RM})$ de encéfalo mostró una lesión hiperintensa en la secuencia de FLAIR en la región anterior de la corteza occipital izquierda (Figura 2). La espectroscopia por RM mostró una disminución de los picos de colina, creatina y $\mathrm{N}$-acetil-aspartato, además de un aumento del pico correspondiente al lactato, compatible con un infarto (Figura 3). El



FIgURA 2. Resonancia magnética de encéfalo ponderada en FLAIR. Se observa una lesión hiperintensa en la región anterior de la corteza occipital izquierda. estudio etiopatogénico del infarto fue negativo (Tabla 1). Se le indicó aspirina en dosis de 325 $\mathrm{mg} /$ día y se encuentra estable 6 meses después de su consulta. La RM y la espectroscopia por RM de control a los 6 meses no mostró cambios respecto a la inicial.

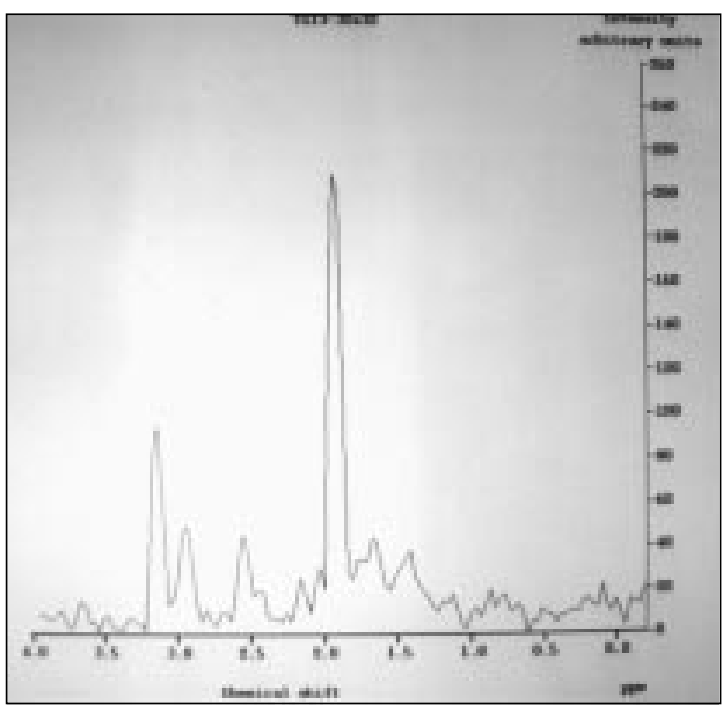

FigURA 3. Espectroscopia por resonancia magnética. En las curvas 1, 2 y 3, el voxel se localizó en la lesión occipital, la curva 4 corresponde al control normal en la región occipital contralateral. Se observa una disminución de los picos de colina, creatina y Nacetil-aspartato y un aumento del pico de lactato en las curvas 1,2 y 3 , lo que sugiere una lesión isquémica ${ }^{12}$. 
Tabla 1. Estudio etiopatogénico del infarto occipital (todos en rangos normales)

Angiorresonancia intra y extracraneana

Ecocardiograma transesofágico

Holter de arritmias de 24 horas

Ecografía Doppler-Dúplex de arterias

carótidas y vertebrales

Hiperhomocisteinemia

Déficit de antitrombina III

Déficit de proteína $C$
Déficit de proteína $\mathrm{S}$

Resistencia a la proteína $\mathrm{C}$ activada

Factor V Leiden

Mutación de la protrombina G20210A

Anticoagulante lúpico

Anticardiolipinas

Anti-beta II glicoproteína

\section{DISCUSIÓN}

En 1926, Brouwer y Zeeman demostraron, en animales de experimentación, que luego de la destrucción de la retina nasal periférica, se producía la degeneración de un haz de fibras ubicadas en la porción medial del nervio óptico, que continuaba en el quiasma hacia el tracto óptico y luego se extendía al cuerpo geniculado lateral contralateral ${ }^{1}$. El mismo año se descubrió que este haz sólo contenía fibras contralaterales ${ }^{2}$. En 1932, se confirmó este hallazgo y se postuló que "a la porción más anterior de la corteza estriada, se proyectan sólo las fibras de la retina nasal periférica contralateral"2.

Dado que el campo visual temporal de cada ojo es más extenso que el nasal ( $30^{\circ}$ más), las fibras correspondientes al campo temporal más periférico (entre los $60^{\circ}$ y $90^{\circ}$ ) de cada ojo, no tienen correlación con las del campo nasal del ojo contralateral en la corteza occipital ${ }^{3,4}$. Es el daño de estas fibras no representadas en la retina del ojo contralateral, el que produce un defecto con forma de creciente, cuya parte más ancha se encuentra en el meridiano horizontal y que se extiende entre los $60^{\circ}$ y $90^{\circ}$ externos. Lesiones localizadas en la corteza visual primaria, delimitadas sólo a estas fibras no pareadas provenientes de la retina nasal del ojo contralateral, originan el síndrome del creciente temporal o de la media luna ${ }^{5,6}$.

Comunicaciones de este síndrome son infrecuentes. Bender y Strauss publicaron una serie de 10 pacientes con un defecto de creciente temporal en un estudio de 100 enfermos con tumores cerebrales que comprometían la radiación óptica ${ }^{7}$.
Walsh publicó el caso de un paciente con pérdida del creciente temporal monocular, sin determinar su etiología ${ }^{8}$. Landau et al, reportaron dos pacientes con este síndrome: uno con una malformación arteriovenosa extensa a nivel temporal y otro con una lesión cortical occipital de etiología no determinada $^{9}$. Chavis et al, publicaron el caso de otra paciente con una lesión de la corteza estriada que tampoco fue estudiada ${ }^{10}$. Nuestro paciente presentaba una lesión occipital contralateral en la región más anterior de la corteza estriada, la RM y la espectroscopia por RM establecieron que la lesión era isquémica (Figuras 2 y 3). El estudio etiológico del infarto fue negativo (Tabla 1).

Ante un paciente con un síndrome de la media luna se debe tener dos consideraciones: Primero, la mayoría de los defectos monoculares corresponden a daños retinianos, así, se deberá examinar minuciosamente la retina nasal periférica ${ }^{2}$. Segundo, dado que este defecto comienza en los $60^{\circ}$ laterales, para detectarlo se debe realizar un examen detallado de campo visual, ya sea con perímetro de Goldmann o clínicamente, aproximando un objeto al paciente desde posterior y lateral. Dado lo periférico del área afectada (entre los $60^{\circ}$ y $90^{\circ}$ laterales), y que la perimetría computarizada automatizada sólo explora los $30^{\circ}$ alrededor del punto de fijación es del todo probable que este déficit pase desapercibido en este examen ${ }^{11}$.

En conclusión, presentamos el caso de un paciente con un infrecuente síndrome neurooftalmológico, el síndrome del creciente temporal o de la media luna. Su reconocimiento debe plantear una lesión cortical occipital, específicamente en la región más anterior de la corteza calcarina contralateral. 


\section{REFERENCIAS}

1. Brouwer B, ZeEman WPC. The projection of the retina in the primary optic neuron of monkeys. Brain 1926; 49: 1-35.

2. MiLer NR, Newman NJ. Topical diagnosis of lesions in the visual sensory pathway. En: Miller NR, Newman NJ. (eds). Walsh and Hoyt's Clinical Neuro-Ophtalmology. Baltimore. Williams and Wilkins 1998; 237-386.

3. Benton S, Levy I, Swash M. Vision in the temporal crescent in occipital infarction. Brain 1980; 103: 83-97.

4. McFadzean R, Brosnahan D, Hadiey D, Mutlukan E. Representation of the visual field in the occipital striate cortex. Br J Ophthalmol 1994; 78: 185-90.

5. Ask-Upmark E. On the cortical projection of the temporal half-moon of the visual field. Acta Ophthalmol 1932; 10: 271-90.

6. Cole M. When the left brain is not right the right brain may be left: report of personal experience of occipital hemianopia. J Neurol Neurosurg Psychiatry 1999; 67: 169-73.

7. Bender MB, StRauss I. Defects in visual field of one eye only in patients with a lesion of one optic radiation. Arch Ophthalmol 1937; 17: 76587.

8. WALSH TJ. Temporal crescent or half-moon syndrome. Ann Ophthalmol 1974; 6: 501-5.

9. Landau $\mathrm{K}, \mathrm{WichmanN} \mathrm{W}$, Valavanis $\mathrm{A}$. The missing temporal crescent. Am J Ophthalmol 1995; 119: 345-9.

10. Chavis PS, Al-Hazmi A, Clunie D, Hoyt WF. Temporal crescent syndrome with magnetic resonance correlation. J Neuroophthalmol 1997; 17: 151-5.

11. LEPORE FE. The preserved temporal crescent: the clinical implications of an "endangered" finding. Neurology 2001; 57: 1918-21.

12. López M, Huete I. Espectroscopia por resonancia magnética en neurología. "La biopsia no invasiva". Cuad Neurol 2002; 26: 53-63. 